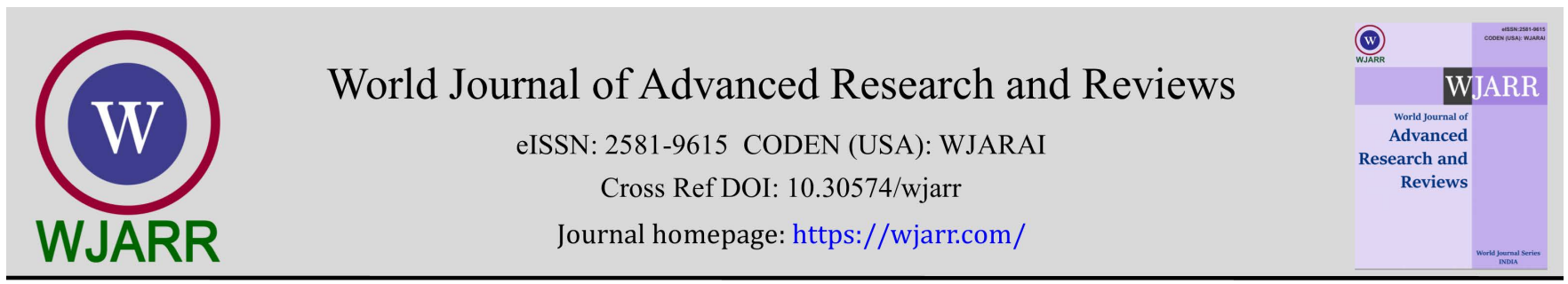

(REVIEW ARTICLE)

\title{
Understanding psychosis: treatment and rehabilitation (updates for clinicians)
}

\author{
Gayane Kirakosyan * and Alina Frolova \\ Regional Referral Psychiatric Hospital, Odesa, Odesa Region, Ukraine.
}

World Journal of Advanced Research and Reviews, 2022, 13(01), 115-121

Publication history: Received on 02 December 2021; revised on 04 January 2022; accepted on 06 January 2022

Article DOI: https://doi.org/10.30574/wjarr.2022.13.1.0006

\begin{abstract}
Psychosis is a group of psychotic disorders. Its manifestation depends on the specific type of functional violation. However, this is characterized by a gradual increase in clinical signs and a change in behavior. Symptoms of psychosis can be recognized by the following manifestations: hallucinations, delusional ideas, movement disorders, mood disorders including manic and depressive disorders and changes in emotional sphere. Psychosis occurs due to problems in the functioning of neurons. Due to the violation of bonds in the molecules, they do not receive nutrition and they are deficient in oxygen. This leads to the fact that neurons cannot transmit nerve impulses; multiple dysfunctions occur in the central nervous system. The type of psychosis depends on a part of the brain suffered from the hunger strike. The causes of this disorder are of 3 types: endogenous, associated with internal processes, exogenous or external and organic, when the causes of psychosis are changes in the brain such as tumors, trauma or hemorrhage. Psychosis is usually treated in a hospital setting. Such patients require urgent admission as they cannot control their actions, they can harm themselves and others. Psychosis is a relapse-prone disease. With timely and comprehensive treatment, the prognosis will be favorable. This review article is a good educational material for medical and psychological practitioners whose goal is to improve knowledge of treatment and rehabilitation processes of psychosis and its related disorders.
\end{abstract}

Keywords: First Episode Psychosis; FEP; Antipsychotic; Psychosis Treatment; Psychosis Rehabilitation

\section{Introduction}

Psychosis is a state that affects the mind, in which it is difficult for people to distinguish between what is real and what is not. This can include conditions in which visions or sounds occur that other people cannot see or hear (hallucinations), or believe that is not true (delusions) [1-3]. The combination of hallucinations and delusional thinking can lead to severe distress and behavioral changes. The first episode of psychosis is the first time a person experiences an episode of psychosis (FEP). The nature of symptoms in the prodromal period of FEP remains unclear. Different causes, including combination of a person's genetics and life experiences, stressful events, substance use or even physical health conditions (seizure disorders, cognitive impairments, Parkinson's disease and damage to the brain from interruption of its blood supply) can cause psychosis [4-9]. Many people recover from the FEP and never experience another psychotic episode. Early intervention teams specialize in treating early psychotic episodes and strive to treat it as quickly and intensively as possible [10]. Intensive and early treatment of psychosis can help more people continue advance treatment, rehabilitation and recover. After early treatment, the person will be managed by their family doctor or mental health professionals. However, it is important to remember that psychosis can be a sign of a mental condition, such as psychotic spectrum disorders (delusional disorder, schizophrenia, schizoaffective disorder) and mood spectrum disorders (bipolar disorder or major depressive disorder) [10-16]. Correct treatment begins with correct diagnosis. Sometimes symptoms that are mistakenly attributed to the onset of psychosis are actually manifestations of very different diseases. In such cases, subsequent failures in treatment are naturally explained by the initially incorrect

\footnotetext{
* Corresponding author: Gayane Kirakosyan

Regional Referral Psychiatric Hospital, Odesa, Odesa Region, Ukraine.
}

Copyright (C) 2022 Author(s) retain the copyright of this article. This article is published under the terms of the Creative Commons Attribution Liscense 4.0. 
diagnosis. Depending on the patient's situation and condition, treatment may include psychopharmacology, psychotherapy, occupational therapy, physiotherapy and complex rehabilitation.

\section{Basis of psychosis treatment}

It is important to understand exactly when an unstable state occurs, which can subsequently lead to serious consequences for patient's health. The criterion for any disease (not necessarily mental) is maladjustment. So, in a person experiencing stomach pains, performance decreases, mood worsens, the need to communicate with friends disappears and eventually they cease to fully perform their social role. The same can be said about mental illness. The first symptom of the disorder is social maladjustment: there is a sharp change in lifestyle, a change of interests, ignorance of household and work responsibilities, ridiculous statements and actions. Any acute psychosis goes through several stages, which are not always noticed by the patient's relatives. As a rule, it all starts with disturbance of night sleep, anxiety, decreased or inadequately elevated mood and disturbance of food intake [18]. During this period, the patient can focus on issues that did not interest them before, give events a special meaning [19-20]. As the disease progresses, hallucinations are connected to the experiences: the patient hears non-existent voices, feels the change in the world around, begins to feel surveillance and influence from the outside, as if someone is controlling their thoughts, sees special "signs" around. These experiences are accompanied by intense feelings of fear. There is no need to hope that everything will pass by itself. Quite often in the practice of a psychiatrist, there are cases of suicide or injury to another person under the influence of psychotic experiences. Speaking about the nature of the disease, it is necessary to clarify what exactly happens in the human brain at this moment. It is known that the fault is in the imbalance of neurotransmitters that are involved in the transmission of nerve impulses to the cerebral cortex [18]. Similar to depression and other mental disorders, when more or less neurotransmitters become inadequate, the human brain begins to work incorrectly [13-14, 18, 20-22]. It is possible to return the balance to its place, in the first days of an adequate therapy, only with the help of psychopharmacology, subsequently psychological and psychotherapeutic assistance and relevant support of patient's relatives [18, 20, 23].

Psychosis, as a rule, requires admission so that the patient cannot harm themselves and those around them. The rehabilitation course includes drug therapy along with psychological help. In addition, physiotherapy is used, which relieves emotional stress and psychoeducational programs that develop a positive outlook on the world around the person. Timely start of treatment of psychosis is a guarantee of an early return to patient's usual way of life and the prevention of irreversible mental disorders.

Treatment of psychosis is carried out with the help of special drugs - neuroleptics (antipsychotics), as well as antidepressants and mood stabilizers [8, 11-13, 18, 23-25]. Usually, physician chooses treatment strictly individually, taking into account the peculiarities of the manifestations of the disorder, the patient's state of health and a number of other factors. An attempt to self-control the state of psychosis can lead to disastrous consequences. As a rule, even after the relief of the state of acute psychosis, the patient requires further treatment and long-term observation by a psychiatrist. In a number of cases, the symptoms become chronic, change the patient's personality, which sometimes requires lifelong treatment.

To reduce the risk of an exacerbation of a psychotic disorder, patient should:

- $\quad$ Follow a measured and orderly lifestyle;

- $\quad$ Do healthy and balanced physical activity;

- Follow a balanced diet and avoid alcohol and other substances;

- Regular compliant use of prescribed maintenance therapy;

Any changes in the usual rhythm of wakefulness and sleep can lead to a relapse. The first signs of relapse are poor appetite, insomnia and extreme irritability. Such signs require examination of the patient by the attending physician.

\subsection{Complex issues in a treatment of psychosis}

Psychoses tend to recur and patients with this diagnosis require regular preventive monitoring [18, 20]. Therefore, international psychiatric conventions give clear recommendations on the duration of the main treatment as well as preventive and maintenance. Those patients who have had their first episode of acute psychosis need to take small doses of antipsychotics for two years as preventive therapy $[10,18,20]$. If they have a repeated exacerbation, then the period of preventive therapy is increased by 2 to 3 years. With the continuous course of the disorders, supportive therapy is carried out, the terms of which are established by the attending physician. Practicing psychiatrists believe that during the initial hospitalization of a patient with acute psychosis, treatment regimens should be covered as 
extensively as possible and full-fledged, long-term social and psychological rehabilitation measures should be carried out in order to reduce the risk of relapse of the disease.

A person can endure a transient psychotic state caused by taking certain medications and drugs. So-called psychogenic or reactive psychosis may result from the impact of severe mental trauma (stressful situation with danger to life, loss of a loved one, etc.). Often there are so-called infectious (developing as a result of a severe infectious disease), somatogenic (caused by severe somatic pathology, such as myocardial infarction) and intoxication psychoses. The most striking example of the latter is alcoholic delirium or delirium tremens [26]. Even problem gambling is harmful to psychological and physical health. People who live with this addiction may experience depression, migraine, distress, anxiety-related problems and even develop psychosis [26-27]. Psychotic disorders are a very common type of pathology. Statistical data in different regions differ from each other, which is associated with different approaches and possibilities for identifying and accounting for these sometimes difficult conditions for diagnosis. On average, the frequency of endogenous psychoses is $3-5 \%$ of the population [28].

Despite the fact that psychoses are a complex group that includes conditions of various origins, the principles of treatment for them are the same. All over the world, drug therapy is considered the most effective and reliable method of treating psychosis [17-18, 20]. During its implementation, an unconventional, strictly individual approach is applied to each patient, taking into account age, gender and the presence of a history of other diseases. One of the main tasks of a specialist is to establish fruitful cooperation with a patient. It is necessary to instill in the patient faith in the possibility of recovery, overcome their prejudice against the harm caused by psychotropic drugs, convey to them their conviction in the effectiveness of treatment, subject to systematic adherence to prescribed treatment. Otherwise, there may be a violation of medical recommendations regarding doses and medication regimen. The relationship between a doctor and a patient should be based on mutual trust, which is guaranteed by the specialist's adherence to the principles of nondisclosure of information, medical secrecy and confidentiality of treatment. The patient, in turn, should not hide from the doctor such important information as the fact of using psychoactive substances (drugs) or alcohol, taking medications used in general medicine, driving a car or operating complex mechanisms. The female patient should inform the doctor about the pregnancy or breastfeeding of the child. Often, relatives or patients themselves, having carefully studied the annotations to the drugs recommended to them, are perplexed and sometimes indignant, that the patient was prescribed a drug for the treatment of schizophrenia, while they have a completely different diagnosis. The explanation is that almost all drugs used in psychiatry act nonspecifically, i.e. help with the widest range of painful conditions (stress, mood, psychotic, etc.); it is all about the prescribed dose and the doctor's skill in choosing the optimal treatment regimen.

Undoubtedly, taking medications should be combined with social rehabilitation programs and if necessary, with family psychotherapeutic and psychoeducation. Social rehabilitation is a complex of programs for teaching patients with mental disorders the methods of rational behavior both in hospital conditions and in everyday life. Rehabilitation focuses on teaching social skills for interacting with other people, skills needed in everyday life, such as keeping track of your own finances, cleaning your home, shopping, using public transport, professional education, which includes the actions necessary to get and keeping the job, and educating those patients who want to graduate from high school or college. Psychotherapy helps the mentally ill feel better about themselves, especially those who feel inferior to themselves due to their illness and those who seek to deny the presence of illness. Psychotherapy helps the patient to master ways of solving everyday problems. An important element of social rehabilitation is participation in the work of mutual support groups together with other people who understand what it means to be mentally ill. These groups, led by patients who have undergone hospitalization, allow other patients to feel help in understanding their problems, as well as expand their opportunities for participation in recovery activities and social life.

All of these methods, if used judiciously, can increase the effectiveness of drug therapy, but are not able to completely replace drugs. Unfortunately, until now, science does not know ways to cure mental disorders once and for all, psychoses often tend to recur, which requires long-term preventive medication.

\section{Special therapies of psychosis}

The main drugs used to treat psychosis are the so-called neuroleptics or antipsychotics. The first chemical compounds with the property to treat psychosis were discovered in the middle of the last century. Then for the first time in the hands of psychiatrists was a powerful and effective remedy for the treatment of psychosis. Such drugs as chlorpromazine, haloperidol, trifluoperazine and a number of others have proven themselves especially well. They relieved psychomotor agitation well, eliminated hallucinations and delirium. With their help, a huge number of patients were able to return to life, to escape from the darkness of psychosis. However, over time, evidence has accumulated that these drugs, later called classical antipsychotics, affect only positive symptoms, often without affecting negative ones. 
In many cases, the patient was discharged from the psychiatric hospital without delusions and hallucinations, but became passive and inactive, unable to return to work. In addition, almost all classical antipsychotics cause extrapyramidal side effects (especially drug-induced parkinsonism). These effects are manifested by muscle stiffness, tremors and convulsive twitching of the limbs, sometimes a difficultly tolerated feeling of restlessness appears, due to which the patients are in constant motion, unable to stop for a minute. To reduce these unpleasant phenomena, doctors are forced to prescribe a number of additional drugs, which are also called correctors (e.g., benzhexol, trihexyphenidyl, amantadine, etc.). The side effects of classical antipsychotics are not limited to extrapyramidal disorders; in some cases, there may be salivation or dry mouth, urinary disorders, nausea, constipation, palpitations, a tendency to lower blood pressure and fainting, weight gain, decreased libido, erectile dysfunction and ejaculation, in women, galactorrhea (discharge from the nipples) and amenorrhea (disappearance of menstruation) are frequent. It is impossible not to state side effects from the central nervous system: drowsiness, impairment of memory and concentration of attention, increased fatigue, the possibility of the development of the so-called neuroleptic depression. Psychiatric comorbidities, such as bipolar disorder, depression and ADHD should be treated in accordance with current treatment trends and protocol $[11-12,14,16,18-19,29-30]$.

A real revolution in the treatment of psychotic disorders was the discovery and introduction into clinical practice in the early 90 s of a fundamentally new generation of antipsychotics - atypical antipsychotics. The latter differ from classical neuroleptics in the selectivity of their neurochemical action. Acting only on certain nerve receptors, these drugs, on the one hand, turned out to be more effective, and on the other, much better tolerated. It was found that they practically do not cause extrapyramidal side effects. Currently, there are already several such drugs available worldwide risperidone, olanzapine, quetiapine, aripiprazole and clozapine. All of these drugs are highly effective in various psychotic conditions. However, while most of the drugs are more often prescribed by practitioners in the first place, clozapine is reasonably used only in the absence of an effect from previous treatment, which is associated with a number of pharmacological features of this drug, the nature of side effects and specific complications, which, in particular, require regular monitoring general blood test and comprehensive physical checkup. It is also important to mention that all patients treated with psychosis require detailed and regular dental and medical care and checkups as their general health condition can be significantly deteriorated during the course of psychosis [31-32].

Endogenous disorders are characterized by different durations and tendencies to recurrence. That is why international recommendations regarding the duration of outpatient (supportive, preventive) treatment clearly stipulate its terms. From the common clinical practice, patients who have undergone the first episodes of psychosis as preventive therapy need to take small doses of drugs for one to two years. In the event of a repeated exacerbation, this period increases to 3-5 years. If the disorder shows signs of a transition to a continuous course, the duration of maintenance therapy is extended indefinitely. That is why there is a reasonable opinion among practical psychiatrists that for the treatment of newly ill patients (during their first admission) maximum efforts should be made, as long as possible and a full-fledged course of treatment and social rehabilitation should be carried out. All this will pay off fully if it is possible to save the patient from repeated exacerbations and admissions, because after each episode of psychosis consequences will be difficult to treat.

Reducing the recurrence of mental illness is facilitated by an orderly lifestyle of daily living that maximizes therapeutic benefits and includes regular exercise, reasonable rest, a stable daily routine, a balanced diet, avoiding drugs and alcohol and regularly taking medications prescribed by attending physician as supportive therapy.

During psychopharmacology, great importance is given to the correct selection of the drug: it should have minimal side effects at the minimum dosage that would be suitable for a particular patient. In the treatment of psychosis, doctors should prefer monotherapy (the use of one drug). This can significantly reduce the number of side effects. Unfortunately, it often happens that, unnecessarily, the patient is prescribed several drugs of the same class. Such therapy does not bring the expected positive result, but only, on the contrary, increases the number of side effects, which negatively affects the patient's condition.

The selection of the appropriate dosage of the drug is equally important: the same dose for some patients may be insufficient and ineffective, while for others it may be excessive and causing side effects. To determine the optimal dose of the drug, the concentration of the drug in the patient's blood should be tested. If the drug has active metabolites (degradation products in the body) then the concentration of metabolites is also analyzed.

Psychotherapeutic techniques designed to help the patient understand the fallacy of their delusional ideas.

On an outpatient basis, treatment for psychosis is supportive. For a psychiatrist, the primary task is to motivate the patient to continue treatment, which is facilitated by their psychoeducation. It is a way of communicating information 
about the disorder and its treatment to the patient and their family members. The psychiatrist explains to the patient what can cause an exacerbation, to what it leads. It is important for the patient to know by what signs to determine the deterioration of his condition and how to avoid situations that provoke the onset of an exacerbation.

During the outpatient period of psychosis treatment, the patient visits a psychiatrist to monitor their condition. If the patient has complaints, the doctor can correct the treatment in time: doctor controls the concentration of the drug, which depends on a large number of factors, for example, from taking other drugs or smoking. In some cases, the drug can accumulate in the body, leading to intoxication.

In the event that the patient does not want or simply is not able to systematically take medications, they should be prescribed depot drugs. These are long-acting drugs that allow maintaining the required concentration level of the drug in the patient's body. However, such drugs also have certain disadvantages as although side effects are less frequent, they are much more pronounced and more difficult to correct. In addition, setting the appropriate dose and frequency of drug administration is a complex process that requires long-term follow-up.

Together with drug therapy, a significant place in outpatient treatment is occupied by non-drug methods, the main of which is psychotherapy. It helps to understand the fallacy of delusional ideas and to correct cognitive impairments (impairment of memory, attention, thinking).

Psychotherapy has many varieties: family, individual and group, behavioral, communicative corrective, cognitive. Metacognitive therapy (training) plays an important role during psychosis treatment as this type of therapy targets patient's delusions [33].

The final stage in the treatment of psychosis is rehabilitation. At this stage, the main goal is to return the patient to society, teaching them the skills lost during the illness. Rehabilitation of patients with psychosis takes place in several directions. The main ones are:

- $\quad$ Cognitive rehabilitation: includes trainings of cognitive functions that contribute to the restoration of thinking, attention and memory of the patient;

- $\quad$ Social rehabilitation: in the course of social skills training, patients learn interpersonal communication. It has been proven that in patients with psychosis, emotional recognition disorders are frequent, it is difficult for them to respond in familiar social situations. Special trainings help to correct these violations. Most often it is carried out by role-playing situations. The social skills acquired during the trainings are gradually transferred to life by the patients;

- Occupational (professional) rehabilitation: this is a gradual entry into work, allowing the patient to restore the work skills acquired before the illness. After completing labor rehabilitation, the patient gets the opportunity to return to his usual work activity, whether it is school or work.

Thus, after completing the full course of psychosis treatment, the patient can return to a fulfilling life.

\section{Conclusion}

Psychosis is a disorder of mental activity when the sick person gives out mental reactions that do not correspond to external stimuli. Their perception of the situation changes greatly and their behavior is highly disorganized. With psychosis, the patient's basic mental processes such as perception, memory, attention and thinking are disturbed. Psychosis is manifested by painful reactions such as delusions, hallucinations, depersonalization. Psychosis is a clearly expressed disorder of mental activity, in which mental reactions grossly contradict the real situation. This disorder can be caused by a wide variety of reasons such as schizophrenia, intoxication, use of psychoactive substances and alcohol, brain injuries and tumors and psychogenic causes. In any case, a psychotic state requires immediate medical and psychological actions. It is necessary to establish the cause of the psychosis and organize the correct treatment. During psychopharmacotherapy, great importance is attached to the rational and adequate medication selection: it should have minimal side effects at the minimum dosage that would be suitable for a particular patient. In the treatment of psychosis, it is important to apply monotherapy. This can significantly reduce the number of side effects. Specialized psychotherapeutic techniques are used to help the patient understand the fallacy of their delusional ideas. The final stage in the treatment of psychosis is rehabilitation. At this stage, the main goal is to return the patient to society, teaching them the skills lost during the illness. 


\section{Compliance with ethical standards}

\section{Acknowledgments}

We thank our supervisors for their support during the preparation process of this manuscript.

\section{Disclosure of conflict of interest}

The authors declare that there is no conflict of interest.

\section{References}

[1] Tsarkov A, Patrick M, Petro P. Uncommon presentation: Folie à deux (Case study). World Journal of Advanced Research and Reviews (WJARR). 2020; 6: 43-49.

[2] Arciniegas DB. Psychosis. Continuum: Lifelong Learning in Neurology 21.3 Behavioral Neurology and Neuropsychiatry. 2015; 715.

[3] Kirakosyan G, Frolova A. Delirium (Clinical Presentation, Diagnosis and Treatment): Updates for Neurologists and Psychiatrists. 2020.

[4] Tsarkov A, Msoni P, Petlovanyi P. Induced Delusional Disorder: A Case Report. British Journal of Medical and Health Research. 2018; 12-22.

[5] Lautenschlager NT, Förstl H. Organic psychosis: insight into the biology of psychosis. Current psychiatry reports. 2001; 3(4): 319-325.

[6] Tsarkov A, Petlovanyi P. Use of pramipexole in neuropsychiatry. World Journal of Advanced Research and Reviews (WJARR). 2020; 7(2): 82-88.

[7] Lebedyn Z. Diagnostic Procedure and Therapeutic Approaches in Nonconvulsive Status Epilepticus (NCSE). Origins (toxic/dysmetabolic, or of mixed etiology). 2020; 15: 18.

[8] Tsarkov A, Petlovanyi P. Neuropsychiatric aspects of a common problem: stroke. European Journal of Medical and Health Sciences (EJMED). 2019; 1(3): 1-6.

[9] Tsarkov A, Petlovanyi P, Paul R, Prashar L. Modern approach to the treatment of Parkinson's disease: the role of pramipexole in the correction of motor and non-motor disorders. British Journal of Medical and Health Research (BJMHR). 2017; 4(2): 63-71.

[10] Fusar-Poli P, McGorry PD, Kane JM. Improving outcomes of first-episode psychosis: an overview. World psychiatry. 2017; 16(3): 251-265.

[11] Tsarkov A, Petlovanyi P. Bipolar Disorder in Child Psychiatric Practice: A Case Report. Medical Journal of Zambia. 2016; 43(1): 41-46.

[12] Tsarkov A, Petlovanyi P. Bipolar disorder in child psychiatric practice. The African Digital Health Library (ADHL). 2016.

[13] Hartley S, Barrowclough C, Haddock G. Anxiety and depression in psychosis: a systematic review of associations with positive psychotic symptoms. Acta Psychiatrica Scandinavica. 2013; 128(5): 327-346.

[14] Tsarkov A, Petlovanyi P. Depressive Disorder in Child Psychiatric Practice: A Case Report. The Health Press (Zambia). 2017; 9-16.

[15] Krabbendam L, Myin-Germeys I, De Graaf, R, Vollebergh W, Nolen WA, Iedema J, Van Os J. Dimensions of depression, mania and psychosis in the general population. Psychological medicine. 2004; 34(7): 1177-1186.

[16] Tsarkov A, Petlovanyi P. Depressive Disorder in Child Psychiatric Practice: A.

[17] Hjern A, Lindblad F, Vinnerljung B. Suicide, psychiatric illness, and social maladjustment in intercountry adoptees in Sweden: a cohort study. The lancet. 2002; 360(9331): 443-448.

[18] Petlovanyi P, Tsarkov A. Child Schizophrenia: Theory and Practice. European Journal of Medical and Health Sciences (EJMED). 2020; 2(1): 1-5.

[19] Lebedyn Z. Recommendations For The Management Of Patients With Depression In General Clinical Practice. European Journal of Medical and Health Sciences. 2020; 2(4). 
[20] Yung AR, Yung AR, Pan Yuen H, Mcgorry PD, Phillips LJ, Kelly D, Buckby J. Mapping the onset of psychosis: the comprehensive assessment of at-risk mental states. Australian \& New Zealand Journal of Psychiatry. 2005; 39(11-12): 964-971.

[21] Lebedyn Z. The Use of Antidepressants by General Practitioners and Psychiatrists (Personal Experience). International Journal of Science and Research (IJSR). 2019.

[22] Lebedyn Z. Antidepressants In General Practice And Psychiatry. European Journal of Medical and Health Sciences. 2020; 2(3).

[23] Tsarkov A, Petlovanyi P. The Role of Lamotrigine in the Treatment of Bipolar Depression. Imperial Journal of Interdisciplinary Research (IJIR). 2017; 3(8): 131-134.

[24] Konyushok M. Why Neurologists Should Remember About Antidepressants. Bipolar disorder. 2020; 2(4).

[25] Konyushok M. Autism Spectrum Disorder in Adult Patients: Clinical Presentation And Management. Psychopathology. 2020; 2(4).

[26] Schuckit MA. Recognition and management of withdrawal delirium (delirium tremens). New England Journal of Medicine. 2014; 371(22): 2109-2113.

[27] Tsarkov A, Petlovanyi P. Pathological Gambling: The Old Problem of the Modern World. Imperial Journal of Interdisciplinary Research (IJIR). 2017; 3(8): 216-221.

[28] James SL, Abate D, Abate KH, Abay SM, Abbafati C, Abbasi N, Briggs AM. Global, regional, and national incidence, prevalence, and years lived with disability for 354 diseases and injuries for 195 countries and territories, 19902017: a systematic analysis for the Global Burden of Disease Study 2017. The Lancet. 2018; 392(10159): 17891858.

[29] Petlovanyi P, Tsarkov A. Practical guide and some recommendations for the diagnosis and management of Attention deficit hyperactivity disorder (ADHD). World Journal of Advanced Research and Reviews (WJARR). 2020; 6(3): 257-261.

[30] Tsarkov A, Petlovanyi P. Omega-3 Fatty Acids as an Alternative Treatment for Children with Attention Deficit Hyperactivity Disorder. Imperial Journal of Interdisciplinary Research (IJIR). 2017; 3(2): 1378-1380.

[31] Moreno C, Nuevo R, Chatterji S, Verdes E, Arango C, Ayuso-Mateos JL. Psychotic symptoms are associated with physical health problems independently of a mental disorder diagnosis: results from the WHO World Health Survey. World Psychiatry. 2013; 12(3): 251-257.

[32] Phiri C, Tsarkov A, Petlovanyi P, Lingenda. Factors Contributing To Oral Diseases and Treatment Needs amongst Mental Patients at Chainama Hills College Hospital, Lusaka, Zambia. Imperial Journal of Interdisciplinary Research (IJIR). 2017; 3(11): 495-504.

[33] Wells A. Advances in metacognitive therapy. International Journal of Cognitive Therapy. 2013; 6(2): $186-201$. 